\title{
Generating Street Center-lines from Inaccurate Vector City Maps
}

\author{
Federico Thomas \\ Institut de Robòtica i Informàtica Industrial (CSIC - UPC) \\ Gran Capità 2, 2 planta \\ 08034 Barcelona \\ SPAIN \\ e-mail: fthomas@iri.upc.es \\ Tel.: +34934015783 \\ Fax: +34934015750
}




\begin{abstract}
This paper addresses the problem of automatically generating city street networks from a possibly inaccurate vector description of their blocks. The street network, represented as a graph structure with attributes related to both streets and crossings, is a fundamental data structure for a large number of applications including urban planning and transport management.

The considered inaccuracies derive from the typical errors introduced during hand digitizing; that is, undershooting, overshooting, stacking, and layer misplacing. Extracting the street net when all these possible errors are present is not an easy task because of the huge amount of particular cases to be considered.

The presented algorithm is based on the translation of the vector information directly into a run-length encoded binary image. Then, corrections of the above errors and the street net extraction itself can be expressed in terms of well-known simple morphological operations. In order to attain low computational costs, the key idea has been to carry out these operations directly on the encoded image. The result is an algorithm faster than expected, easy to implement because of the elimination of the particular cases and, as a consequence, with more general applicability.
\end{abstract}




\section{Introduction}

Nowadays, maps are frequently obtained by processing terrain images from satellite vision systems. Not only hand digitizing but also image processing and pattern recognition techniques have been used to extract different kinds of information from these aerial photographs for geological or topographical purposes (Pike 1992). This information lies at the base of most Geographic Information Systems (GIS), i.e. systems that are both databases designed to work with data referenced by spatial or geographic coordinates, as well as a set of procedures for analyzing these data (Star and Estes 1990).

GIS are widely used in different areas, including military applications, environmental studies, geological exploration, etc. They have been proved of particular interest in town mapping where a huge amount of structured data including street names, numbers, special site locations, etc., have to be linked to particular geometric entities. We will focus on this kind of maps.

City maps are usually obtained by standard manual digitizing aerial photographs. One of the major tasks of this labor-intensive work consists of digitizing the road sides and storing the result in a particular layer linework. Utility companies use this information and insert their own distribution networks and related information on separate layers.

The algorithm discussed herein automatically extracts the street network - which is seldom directly available - from the road layer. This information, represented as a graph structure with attributes related to both streets and crossings, is a fundamental data structure for a large number of applications of the aforementioned utility companies.

\subsection{On the difficulty of the problem}

It seems to be an easy task to decide where streets are running along by simply inspecting where city blocks are placed. In practice this becomes a hard task because of two main reasons: (a) vector drawing representations are not unique; and (b) they are, in general, neither accurate nor complete. Below, we present a brief discussion of problems associated with street network automation.

Vector representations have great advantages when working with town maps. They certainly reduce storage costs, simplify scaling, and ease geometric structure modifications. Nevertheless, their main drawback results from the fact that completely different vector drawings, that is, with very different underlying geometric entities, lead to the same graphical appearance. These differences are often due to errors in digitizing, as explained below. For example, the triangle in fig. 1 can be represented using a three-sided polygon, a polyline, and a set of possibly overlapping segments, all of them providing the same appearance on the screen. This is one of the reasons why algorithms for automatic drawing interpretation have focussed on raster representations (Boatto et al. 1991), (Ablameiko and Frantskevich 1992), (Yamada et al. 1993), and hybrid systems combining both raster and vector representations have been used to balance benefits (Jager 1992).

Although hand digitizing leads to drawings that might seem a fair vector representation of the original, a closer view shows a lot of unexpected situations: some geometric primitives are duplicated, overlapping occurs surprisingly often, many intersections between elements are not explicitly represented, and structured elements - such as squares or triangles - are multiply represented by partially overlapped primitive elements. In what follows, data automation errors are classified in one of the following categories: undershooting, overshooting, stacking, and layer misplacing. Fig. 2 contains some examples. 
Undershooting and overshooting are related to the lack of accuracy in line drawings involving polylines: two polylines that should appear connected at their endpoints, actually appear either separated by a given distance (undershooting), or slightly intersecting (overshooting). As a consequence, in our case, city blocks seldom appear as perfect closed shapes. Existing fixing procedures, such as those developed for Intergraph's MicroStation by third parties, lack welldefined procedures and are only able to handle situations on a case by case basis.

Stacking is related with duplication of data. When geometric entities overlap partially or completely, it is said that staking occurs. In general, stacking leads to redundant information and thus a vector representation containing staking errors can be simplified to another equivalent vector representation with no redundancies.

Layer misplacing occurs when there are entities wrongly assigned to a layer. Problems may arise when these wrongly assigned entities modify the actual shape of objects, such as blocks, making their recognition difficult. In our case, segments of sidewalks, internal block walls, etc., may appear in the same layer as blocks.

\subsection{Previous approaches}

In the presented context it should be clear that extracting the street center-lines of a town map is not an easily automatizable task. This is why it is usually carried out either by standard manual digitizing or by center-lining using a CAD environment. Obviously, standard manual digitizing is labor-intensive and, due to the generally small distance between road sides, resulting center-lines may not be strictly parallel to the corresponding road sides. Center-lining, as in the center-line option under the add arc command in ARC-EDIT module using ARC/INFO, as pointed out in (Olson 1995), eliminates this non-parallelism problem and leads to good results if used properly. It is also less tedious than table digitizing but still labor intensive.

Two automated approaches have been proposed up-to-date: the strictly vector approach, and the rasterization-skeletonization-revectorization approach.

From the point of view of the quality of results, the evaluation of any automated approach must be based on its ability to preserve the geometric and topological properties of the street net. Preserving the geometric properties implies that the center-lines are clean, without spurs, medial axes of the original street net. Preserving topological properties (sometimes called homotopy preservation) implies that the center-lines preserve the connection of the net without disconnections and additional loops.

The strictly vector approach is essentially based on the generation of a table of segments perpendicular to discrete points on the street sides. Then, these segments are intersected with all other street sides and those that are not close to perpendicular or are not the first intersection on the ray going out from the center point of the perpendicular segment are eliminated. A proper connection of midpoints of the segments thus obtained is assumed to provide a good approximation of the street center-lines. This approach has been recognized to be specially difficult to implement: several tables have to be used and a great deal of complex indexing is involved (Olson 1995). Moreover, the result is not always successful in the terms given above: in general poor or erroneous description of crossings is obtained, especially when five or more streets meet, and problems occur where paired road side sections deviate significantly from parallel.

On the other hand, the rasterization-skeletonization-revectorization consists of obtaining a raster version of the vector map, applying a skeletonization algorithm to the street areas, and revectorizing the result. This approach provides good results, but its use is limited by its 
low speed and its inability to handle large, or even medium-sized, maps with limited memory. It has been also pointed out that this approach is also troubled by several artifact problems (Olson 1995), but in practice these problems derive from undershooting or overshooting errors that can be easily overcome by using simple morphological techniques, as implemented in our approach.

\subsection{New approach}

It is clear that all approaches, as used in current state-of-the-art practice, have several weaknesses. The alternative presented herein is based on the direct translation of the vector information into a run-length encoded binary image. Then, corrections of the errors contained in the vector representation and the street net extraction itself can be expressed in terms of well-known simple morphological operations. In order to attain low computational costs and reduced memory requirements, the key idea has been to carry out these operations directly on the encoded image. Then, although the presented approach can be classified within the rasterization-skeletonization-revectorization approach, it does not suffer from its main drawback because of the way the generated raster map is encoded and processed. The result is an algorithm faster than expected, easy to implement because of the elimination of all the particular cases and, as a consequence, with a more general applicability. The algorithm proposed can be divided into the following four steps:

- Run-length encoding;

- segmentation of street areas;

- skeletonization of street areas; and

- topology extraction and vectorization of skeleton elements.

This strategy allows us to correct the errors mentioned in Section 1.1, within the first two steps in a very straightforward way. A detailed description of each step is given in the following sections, including examples of map processing results for different areas of the City of Barcelona.

\section{Run-length encoding}

Given a resolution level, the run-length encoding process performs a translation of the vector map to a binary image by casting a grid over the former and identifying those cells of the grid, or pixels, that are trespassed by the geometric elements in the vector map. Then, any scanning line in the binary image can be viewed as a sequence of alternating trespassed and non-trespassed cells. A run-length code represents intervals of trespassed cells, or runs (Jain 1989). A simple run-length code consists of the start address of each interval, followed by the corresponding end address. See (Brown and Shepherd, 1995) for a description of standard run-length codes. This kind of compression is designed to take advantage of the fact that a cell condition, trespassed or not, will often repeat, which is particularly true when working with line drawings (Cardoner and Thomas 1997a). Then, an image can be seen as a list of lines, each line being a possibly empty list of non-overlapping sorted intervals. 
In our implementation the run-length encoded image is obtained by iteratively ORing, as explained below, an accumulator with a run-length-encoded image obtained by encoding just one vector so that the expanded bit-map is never explicitly computed.

Once a run-length encoded version of a vector drawing is obtained, error treatment dramatically changes. Note that a proper run-length encoding process directly corrects most undershooting errors: at least those in which the size of the gap is less than half the pixel size. Moreover, stacked lines, that is, segments overlapping completely or partially, have no influence in the encoded image. Fig. 3 shows three run-length encoded images, corresponding to the same vector map, for three different resolution levels.

The selection of an appropriate rasterization resolution is just a sampling problem. It should be clear that a resolution twice the minimum road width is the minimum acceptable resolution, or critical resolution, that ensures that the topology of the street network is properly captured without being influenced by typical undershoots. Nevertheless, the generated center-lines using a thinning process as explained in Section 4 - will be biased up to the value of the chosen resolution. In other words, the selection of a resolution close to the critical one may lead to unacceptable bias errors in some applications.

Our experiments with maps from Barcelona show that resolutions ten times the critical one behave well in all cases leading to acceptable bias errors. These experiments also show that resolutions around one hundred times the critical one may lead to situations in which overshoots and undershoots are not distinguished from genuine map features (for example, situations in which undershoots are considered as genuine open paths).

The rasterization resolution taken in fig. $3 a, 3 b$ and $3 c$ is 21,14 and 7 times the estimated critical resolution, respectively. Note that the coarsest the resolution, the smoothest shapes one gets. Morphological skeletons, as the one we describe in Section 4, are known to be highly sensitive to noise: single extra pixels in the shape's border might lead to extra skeletal legs and hence to undesired junctions (Pitas and Venetsanopoulos 1990, p. 193). Thus, the aforementioned smoothing effect reduces the possibility of generating extra skeletal legs. Other operations introduced in further processings, which are summarized in the conclusions, also reduce this possibility.

In order to correct the remaining errors and to extract the street net itself, we apply an extensive class of binary image processing techniques called morphological operations (Matheron 1975; Serra 1982). Morphological operations can be described simply in terms of adding or removing pixels from the binary image according to certain rules which depend on the pattern of the neighboring pixels. The simplest of these operations is erosion and dilation.

Erosion consists of removing any pixel touching another pixel that is part of the background. This removes a layer of pixels from around the periphery of all regions which causes some shrinking of dimensions and may cause a region to break into parts.

Dilation consists of adding any background pixel which touches another pixel that is already part of a region. This adds a layer of pixels around the periphery of all regions which causes some increase in dimensions and may cause regions to merge. It also fills in small holes within regions.

There is a rich literature in the field of Mathematical Morphology in which a specific notation for the operations, discussed in terms of set theory, has been developed (Pitas and Venetsanopoulos 1990). Fortunately, we only need a result that can be intuitively understood: any morphological operation on a binary image can be carried out by repeating a sequence of two operations in which the first one shifts the image in a given direction, and the second one applies a logical operation between the original image and the shifted one (Cardoner and 
Thomas, 1997a). For example, if an image Img is eroded, the result can be expressed as:

$$
\text { Erod }=\operatorname{Img} \cap \operatorname{Img}_{0} \cap \operatorname{Img}_{2} \cap \operatorname{Img}_{4} \cap \operatorname{Img}_{6},
$$

where $\operatorname{Img}_{d}$ stands for the image Img shifted in the $\mathrm{d}$ modulo 8 direction. This direction ranges from 0 to 7 , starting from left-to-right and turning clockwise, as shown in fig. 4 .

If the same image is dilated, the result can be simply expressed as:

$$
\text { Dil }=\operatorname{Img} \cup \operatorname{Img}_{0} \cup \operatorname{Img}_{2} \cup \operatorname{Img}_{4} \cap \operatorname{Img}_{6} .
$$

Thus, dilation and erosion transformations are related in that what one does to the image foreground the other does to the image background. This property allows us to perform the erosion in equation (1) by the following dual dilation that uses unions instead of intersections

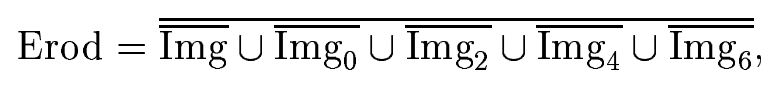

where $\overline{\operatorname{Img}}$ stands for the complement of image Img, that is, its background.

If duality is applied to (2), we get

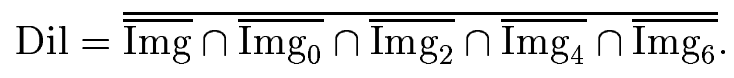

These duality considerations are useful when implementing morphological operations on encoded images to obtain efficient algorithms.

Erosion and dilation are non-commutative operations. Thus, two new operations can be defined upon them: closing, which stands for a dilation followed by an erosion, and opening, for the inverse sequence of operations. In general, a closing operation fills little holes and smoothes shape contours, while an opening operation eliminates isolated points and narrow connections.

A brute-force algorithm to implement shiftings and logic operations on a run-length encoded image would consist of decoding the image, modifying each pixel value, and reencoding the result. Obviously, this should be avoided.

Let us suppose two run-length-encoded images have to be intersected, where $l_{1}$ and $l_{2}$ represent the lists of intervals associated with the same line in the first and second image respectively. Then, the list representing the intersection of both lists of intervals can be computed as follows:

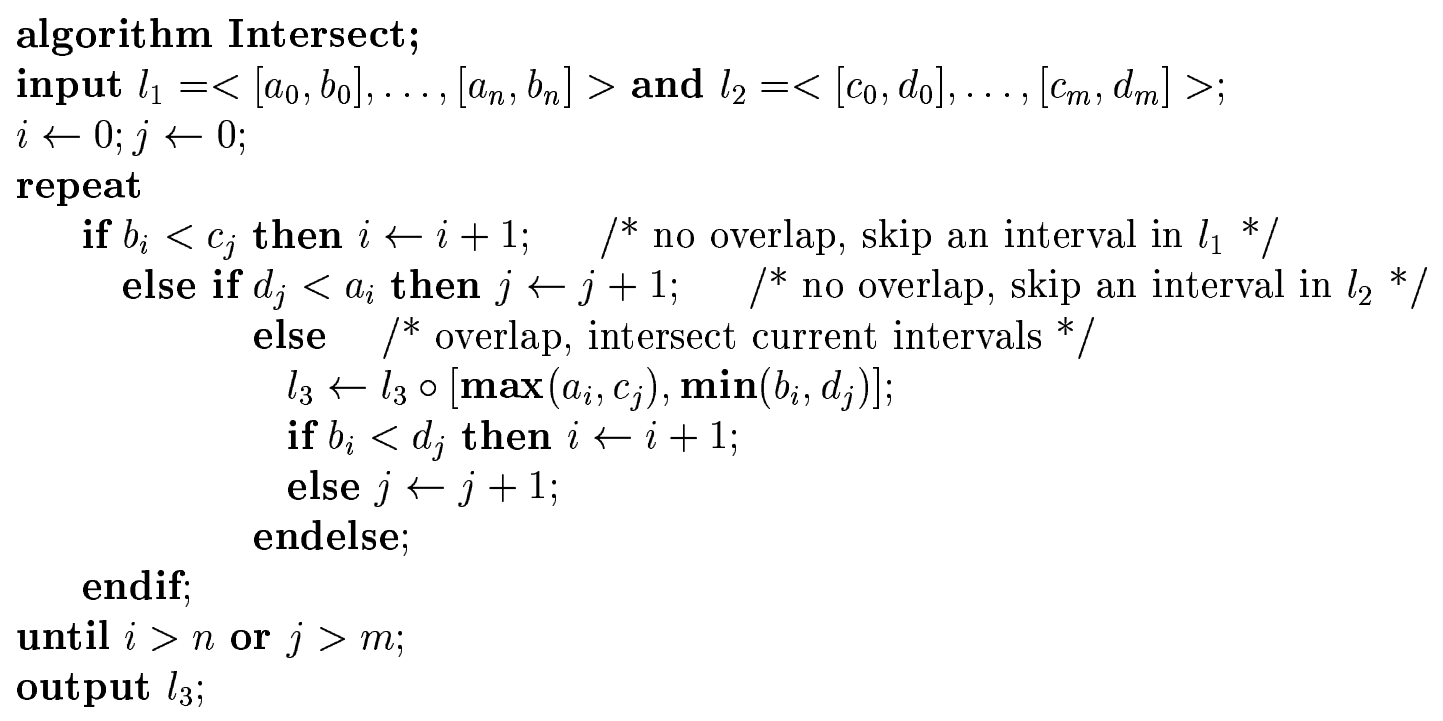


where $\circ$ denotes concatenation. The generated list, $l_{3}$, is computed in linear time in the total input size $\left|l_{1}\right|+\left|l_{2}\right|$.

The algorithm for the union of two lines is similar, and also runs in linear time. Note that both intersection and union operations produce a list $l_{3}$ with at most $\left|l_{1}\right|+\left|l_{2}\right|$ intervals.

By introducing proper indices, the evaluation of shifted images can be easily avoided so that displacements can be taken into account directly when intersecting or unioning images.

After run-length encoding, correction of possibly remaining undershooting errors can be achieved by a simple closing operation. Nevertheless, note that, if an undershooting error is corrected by a closing operation without destroying street topology, a lower resolution in the run-length encoding could initially be taken (if we do not care about bias errors).

The remaining errors, that is overshooting and layer misplacing, will be corrected after region labeling - as it is shown in the next section - based on the simple fact that each city block defines closed shapes on the map, contrary to what usually happens to other features appearing on the map because of layer misplacing. Nevertheless, it is not difficult to imagine situations in which a layer misplacing error cannot be distinguished from a genuine city block. In these cases the result will be obviously incorrect.

\section{Segmentation of street areas}

A run-length encoded version of a vector map without undershooting errors contains a set of closed lines which divides the plane into connected regions. In order to separate these regions the image is first inverted. Then, lines become background and the resulting connected regions in the foreground can be separated using a simple labeling algorithm as explained below. Then, the problem consists of classifying the obtained regions into blocks or streets. In general, while a block area is surrounded by a single street region, a street region surrounds typically more than two blocks. In order to decide which are the labeled regions with more than two surrounding regions, each of them is separately dilated, intersected with the rest and the result, labeled again. The following algorithm implements this simple idea.

\section{algorithm GetStreets;}

input Img; /* image from which to extract streets */

input ImgStreets; /* void image where to accumulate results */

ImgLabel $\leftarrow$ Label $(\overline{\operatorname{Img}})$;

for $(\mathrm{i}=1 ; \mathrm{i}<$ NumLabels(ImgLabel) $; \mathrm{i}=\mathrm{i}+1)$

ImgAux1 $\leftarrow$ Region(i, ImgLabel);

$\operatorname{ImgAux} 2 \leftarrow \operatorname{Img} \cup \operatorname{ImgAux} 1_{0} \cup \operatorname{ImgAux} 1_{2} \cup \operatorname{ImgAux} 1_{4} \cup \operatorname{ImgAux} 1_{6}$;

ImgAux $\leftarrow$ ImgAux2 $\cap$ Img;

${ }^{*}$ All regions connected to the frame are assumed to be a single region */

ImgLabel $\leftarrow$ Label(ImgAux2);

if (NumLabels (ImgLabel2) > 2) then

ImgStreets $\leftarrow$ ImgStreets $\cup$ ImgAux1;

endif;

endfor;

output ImgStreets;

Label, Region and NumLabels have the obvious meaning: Label assigns a different consecutive integer to each connected region in the image (this number appears as an attribute 
to each interval of the corresponding region); Region returns an image containing only the selected region; and NumLabels, the total number of connected regions in the image. Details on how these operations can be implemented for run-length encoded images can be found in (Rosenfeld 1982). Nevertheless, it is worth to mention that the first part of the used labeling algorithm for run-length encoded images detects neighboring intervals in consecutive lines, gives the same label to them, and creates equivalences between labels when required. The second part of the algorithm simply uses these equivalences to generate an image where all the intervals in a connected region have the same label. Figures $5 b$ and $6 b$ show the results of applying this algorithm to the images in figures $5 a$ and $6 a$, respectively.

It is worse to generate false street regions than false blocks. In other words, it is better to obtain an incomplete street net than a wrong one. If the above simple algorithm is used, excellent classification results are obtained. Nevertheless, regions next to the frame may be misclassified, but they always correspond to street regions classified as blocks. Fig. 5c, where this situation arises, shows the classification obtained for the image in fig. $5 b$.

At this stage segments generated by overshooting errors and block walls or sidewalks present in the image because of layer misplacing errors can be directly corrected by performing a closing operation on the classified regions. Fig. $6 d$ shows the result of applying this idea to fig. $6 c$.

The set of street regions are the element to process next. Recall that the final goal is to obtain a representation of the street network described as a graph whose nodes stand for crossings and links, for street segments. To this end, a homotopy-preserving skeletonization processing is used in the next section.

\section{Skeletonization of street areas}

Thinning procedures have been extensively used to obtain skeletons of arbitrary regions for data compression, raster-to-vector conversion and pattern recognition, and as a consequence many different thinning algorithms have been proposed (Jang and Chin 1990). Some of them use a morphological point of view, providing different definitions of binary image skeletons.

In our case, the desired skeleton, besides being one-pixel thick - to ease vectorization - must preserve homotopy - to ensure that the street net topology is properly captured. A skeleton is one-pixel thick when each of its points is necessary to keep connectivity. Then, pixels with more than two neighbors only appear at junctions, i.e. where different skeletal segments meet.

In oder to obtain the desired skeleton, we apply a thinning process consisting of a successive removal of the outer pixels of the regions while retaining those necessary to keep connectivity, until no more progress is made. The following algorithm implements this idea:

\section{algorithm Skeleton}

input: $\mathrm{X}$;

output: $\mathrm{S} ; /^{*}$ skeleton of $X^{*} /$

$S \leftarrow \emptyset$

$A \leftarrow \emptyset$

$S \leftarrow X$

do

$$
\begin{aligned}
& X \leftarrow S \\
& \text { for }(d \leftarrow 0 ; d<8 ; d \leftarrow d+2) \\
& \quad G \leftarrow S \cap \bar{S}_{d} \cap\left[\left(S_{d+1} \cap \bar{S}_{d+2}\right) \cup\left(S_{d-1} \cap \bar{S}_{d-2}\right)\right]
\end{aligned}
$$




$$
\begin{aligned}
& \qquad \begin{array}{l}
E \leftarrow S \cap S_{d} ; \\
R \leftarrow S \backslash\left(E \cup E_{d+4}\right)
\end{array} \\
& \qquad A \leftarrow A \cup R \cup G ; \\
& S \leftarrow A \cup E ; \\
& \text { endfor; } \\
& \text { while }(X \neq S) \\
& \text { end. }
\end{aligned}
$$

This algorithm certainly provides a connected one-pixel thick skeleton, but a formal proof of convergence and homotopy lies outside the scope of this paper. Nevertheless, these theoretical aspects can be found in (Cardoner and Thomas 1997b), where simple modifications of this basic algorithm to increase its efficiency are also described.

Figures $5 d$ and $6 e$ show the result of applying the above algorithm to the street regions of figures $5 c$ and $6 d$, respectively. The resulting street net consists of street segments connecting crossings, and thus an adjacency graph can be easily built as explained in the next section.

\section{Topology extraction and vectorization}

Since the obtained skeleton is one-pixel thick, each pixel has only one or two neighbors unless it is placed at a street crossing. Thus, crossings can be obtained by simply labeling each pixel according to its number of neighbors. Then, the result can be seen as a set of separate street segments and crossings, as shown in fig. 6 e for the street region in fig. $6 d$.

In order to independently treat each street segment, crossings can be removed and the result, labeled. Because of the used run-length format, each street segment can be seen as a list of intervals which can be directly translated into a polyline by properly ordering these intervals. It is clear that only two of these intervals, called terminal intervals, have one of their endpoints disconnected, and two interval endpoints are connected if they are adjacent in two consecutive lines. Then, once a terminal interval is found, it can be placed in the first location of a list and the list can be sorted using the following simple variation of the classical bubble ordering algorithm to obtain the desired polyline:

\section{algorithm VectorizeStreet;}

input $p$;

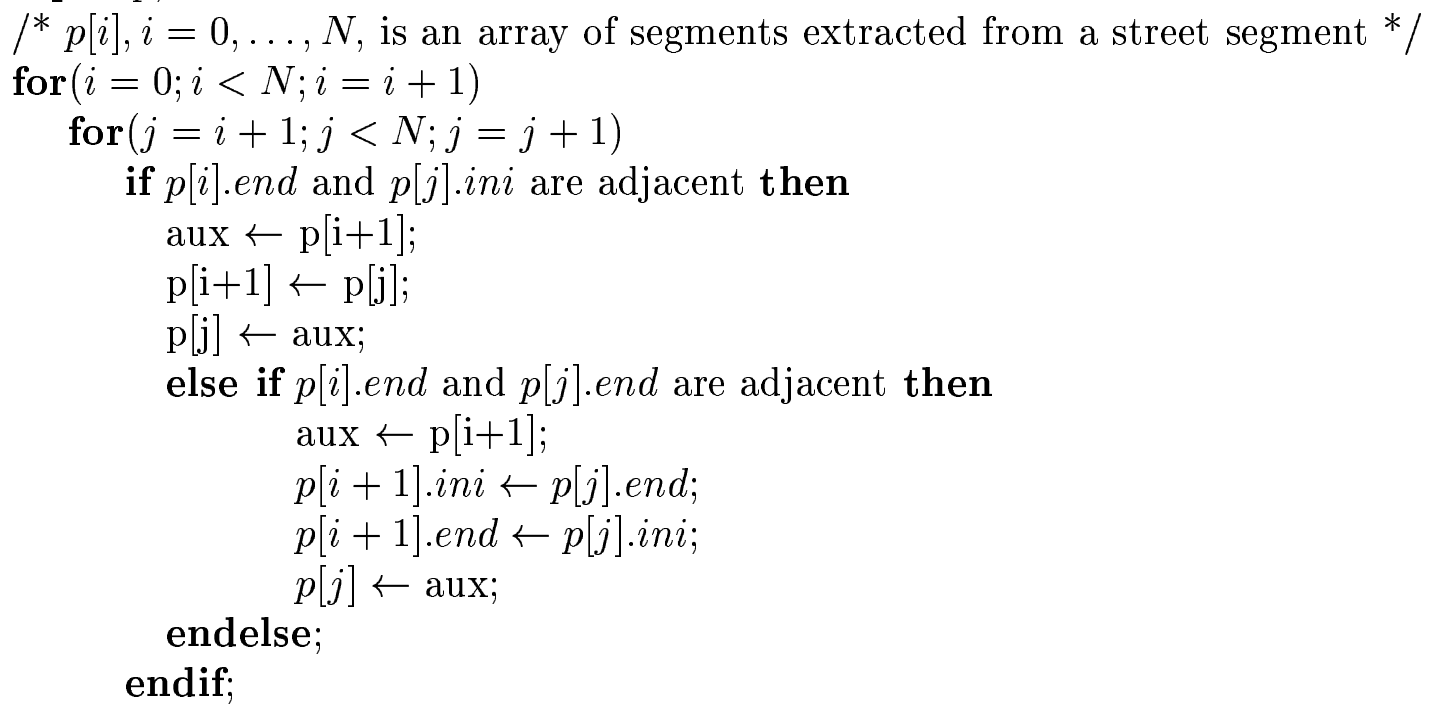


endfor;

endfor;

output $p ; /^{*}$ Now it can be directly interpreted as a polyline */

If the obtained crossings are independently dilated until they overlap a block region, large crossing areas are obtained. If the width of a street connecting two crossings is greater than its length, both crossings overlap after dilation, and it is reasonable to consider the result as a single crossing. The result of applying this idea to one of the examples above is shown in fig. $5 e$. This simplifies the topologic description of the street net. For the skeleton in fig. $5 d$, the initial 48 crossings are reduced to 37 ( $\mathrm{fig} .5 \mathrm{e}$ ), and the number of street segments are reduced from 51 to 42 (fig. $5 f)$. For another example see fig. $6 f$.

In general, the resulting polylines describing the street segments might require further manipulations to reduce the number of points using well-known approximation algorithms (Wall 1982).

Before concluding, let us see the complete sequence of operations for another example. Fig. 7a shows a run-length version of a city map. It contains 7910 intervals and represents a $600 \times 500$ image. Its original vector counterpart in DGN format has 756 polylines, some of them being single segments. First, this image is labeled and segmented, leading to 37 block regions ( $f i g .7 b)$ and one single street region ( $f i g .7 c$ ). A closing operation corrects remaining overshooting and layer misplacing errors $(f i g .7 d)$. Then, after thinning, crossings and street segments are separated leading to 67 crossings and 78 street segments ( fig. 7 e). The final street net, obtained after dilating crossings, contains 53 crossing regions and 60 street segments (fig. 7f). This example takes about 10 seconds on a PC-Pentium (120Mhz) with 16 Mbytes of RAM. Most of this time is devoted to obtain the street region skeleton.

\section{Conclusions}

Geographic Information Systems (GIS) are penetrating a growing number of application domains. Nevertheless, commercially available GIS generally take little advantage of the progress achieved in computational geometry and pattern recognition. They incorporate only a few of the many procedures that have been and are being developed for efficient operations on geometric objects. The incorporation of the algorithm presented herein for automatically generating street center-lines using state-of-the-art morphological processing techniques can be of interest in those GIS dealing with urban planning and transport management.

The developed software package is intended to be used within Intergraph's MicroStation as an MDL application, whose development was sponsored by ENHER, a power utility Spanish company. The algorithm provided a set of street-center lines connected through a set of junctions that correctly represented the topology of Barcelona's street network. Nevertheless, erroneous results were generated because of incorrect description of city blocks (basically, some city blocks were incomplete). These errors were manually corrected and our application, reexecuted. This led to fully successful results for the company's purposes.

The presented examples have been selected among the most pathological areas within the Barcelona city map: they contain a set of serpentine streets with a wide variety of widths so that the street network is far from being a uniform grid, which is usually the case. On seeing the presented results, it is clear that, at some points, the obtained street network description can be done with less crossings and some of the generated crossings are larger areas than 
strictly necessary but, in any case, the topology of the street network was correctly captured and all street center lines properly located. This means that, in order to fit the requirements of a given application, it might be necessary, for example, to eliminate terminal short street segments and crossings connecting only two street segments, or simply connecting generated street center-lines endpoints to the center of mass of the corresponding crossings.

Morphological skeletons are known to be highly sensitive to noise. As mentioned above, a single extra pixel in the shape's border, as mentioned above, might lead to an extra skeletal leg and hence to an undesired junction. The remarkable absence of these artifacts in our results is due to the following facts: (a) generated images are clean without noisy borders because they directly come from vector drawings (they are not the result of a scanning and binarizing process); (b) we use low resolutions, typically ten times the critical one; (c) we perform closing operations on the initial image and on the segmented street region; and (d) we finally dilate crossings.

We wish to stress that, as mentioned above, the main drawback of vector representations results from the fact that completely different vector drawings, that is, with totally different underlying geometric entities, lead to the same visual aspect. This makes vector map processing hard for many applications. In order to overcome this drawback, morphological image processing techniques applied on run-length representations can be of interest for solving many problems arising in map processing. Besides extracting the street net from a city map including different kinds of inaccuracies, as described in this paper, other tasks such as obtaining a simplified description of city blocks free from undershootings and overshootings are appropriate to this kind of processing.

Finally, a point that deserves further attention is the application of morphological shape decompositions for pattern recognition (Pitas ans Sidiropoulos 1992) in large vector maps.

\section{Acknowlegments}

This work has been partially supported by ENHER (Empresa Nacional Hidroeléctrica del Ribagorzana) under several contracts within the frame of the $A L E P H$-CORAL project. I wish also to express my gratitude to J.L. González for his comments and support, and to R. Cardoner for implementing the run-length processing routines.

\section{References}

Ablableyko, S., and Frantskevich, O. (1992). "Knowledge-based technique for mapdrawing interpretation," International Conference on Image Processing and its Applications, No. 354, pp. 550-554, April.

Boatto, L., et al. (1991). "A system for line drawings processing," Proc. of the 6th International Conference on Image Analysis and Processing, Vol. 2, pp. 666-673, September.

Brown, C.W., and Shepherd, B.J. (1995), Graphics File Formats. Reference and Guide, Manning Publications, Greenwich, Connecticut.

Cardoner, R., and Thomas, F. (1997a). "Efficient Morphological Set Transformations on Line Drawings," International Journal of Pattern Recognition and Artificial Intelligence, Vol. 11, No. 6, 1997. 
Cardoner, R., and Thomas, F. (1997b). "Residuals + Directional Gaps = Skeletons," Pattern Recognition Letters, Vol. 18, No. 4, pp. 343-353, 1997.

Jager, E. (1992). "Map up-dating by digital hybrid methods with the SICAD-MAPREVISOR," EARsel Advances in Remote Sensing, Vol. 1, No. 3, pp. 169-172, July.

Jain, A.K. (1989). Fundamentals of digital image processing, Prentice-Hall, Englewood Cliffs, New Jersey.

Jang, B-K., and Chin, R.T. (1990). "Analysis of thinning algorithms using mathematical morphology," IEEE Trans. on Pattern Analysis and Machine Intelligence, Vol. 12, No. 6, pp. 541-551, June.

Matheron, G. (1975). Random sets and integral geometry, John Wiley, New York.

Olson, N. (1995). "An algorithm for generating road center-lines from road rights-of-way," 12th Int. Sym. on Computer-Assisted Cartography, Charlotte, North Carolina, February.

Pike, R. (1992). "Machine visualization of synoptic topography by digital image processing," U.S. Geological Survey Bulletin, Vol. 2016, Chapter B.

Pitas, I. and Venetsanopoulos, A.N. (1990). Nonlinear Digital Filters, Chapter 3, Kluwer Academic Publishers, Boston.

Pitas, I. and Sidiropoulos, N.D. (1991), "Pattern recognition of binary image objects using morphological shape decomposition," Computer vision and image understanding, pp. 279-305.

Rosenfeld, A. and Kak, A.C. (1982). Digital Picture Processing, Academic Press, New York.

Serra, J. (1982). Image analysis and mathematical morphology, Academic Press, London.

Star, J. and Estes, J. (1990). Geographic Information Systems: An Introduction, Prentice Hall.

Yamada, H., Yamamoto, K., Saito, T., and Hosokawa, K. (1993). "Directional mathematical morphology and reformalized Hough transformation for the analysis of topographic maps," IEEE Trans. Pattern Analysis and Machine Intelligence, Vol. 15, No. 4, pp. 380-387, April.

Wall, K. (1986). "Curve fitting based on polygonal approximation," 1986 IEEE Conf. on Img. Proc., pp. 1273-1275, April. 


\section{Figure Captions}

Fig. 1 Vector representations are not unique. A triangle can can be represented, for example, as a three-sided polygon, a polyline, or a set of possibly overlapping segments.

Fig. 2 Vector map containing the four considered errors: undershooting, overshooting, stacking, and layer misplacing.

Fig. 3 Three run-length encoded versions of the vector map in fig. 2 at three different resolutions levels.

Fig. 4 Defined directions on the digital plane.

Fig. 5 (a) Original image used as example; (b) labeled regions where each connected region is represented by a different gray level; (c) segmented street regions including one misclassification next to the frame; (d) obtained skeleton; (e) dilated crossings; and (f) final street net.

Fig. 6 (a) Original image; (b) labeled regions; (c) segmented street region; (d) result after closing to correct overshooting and layer misplacing errors; (d) skeleton; and (f) final street net.

Fig. 7 Another example. (a) Original image; (b) labeled regions; (c) street region; (d) result after closing; (e) skeleton; and (f) final street net. 

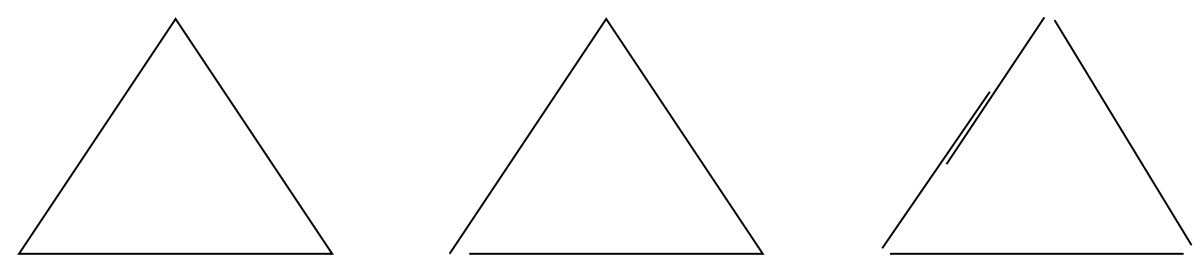

Fig. 1 


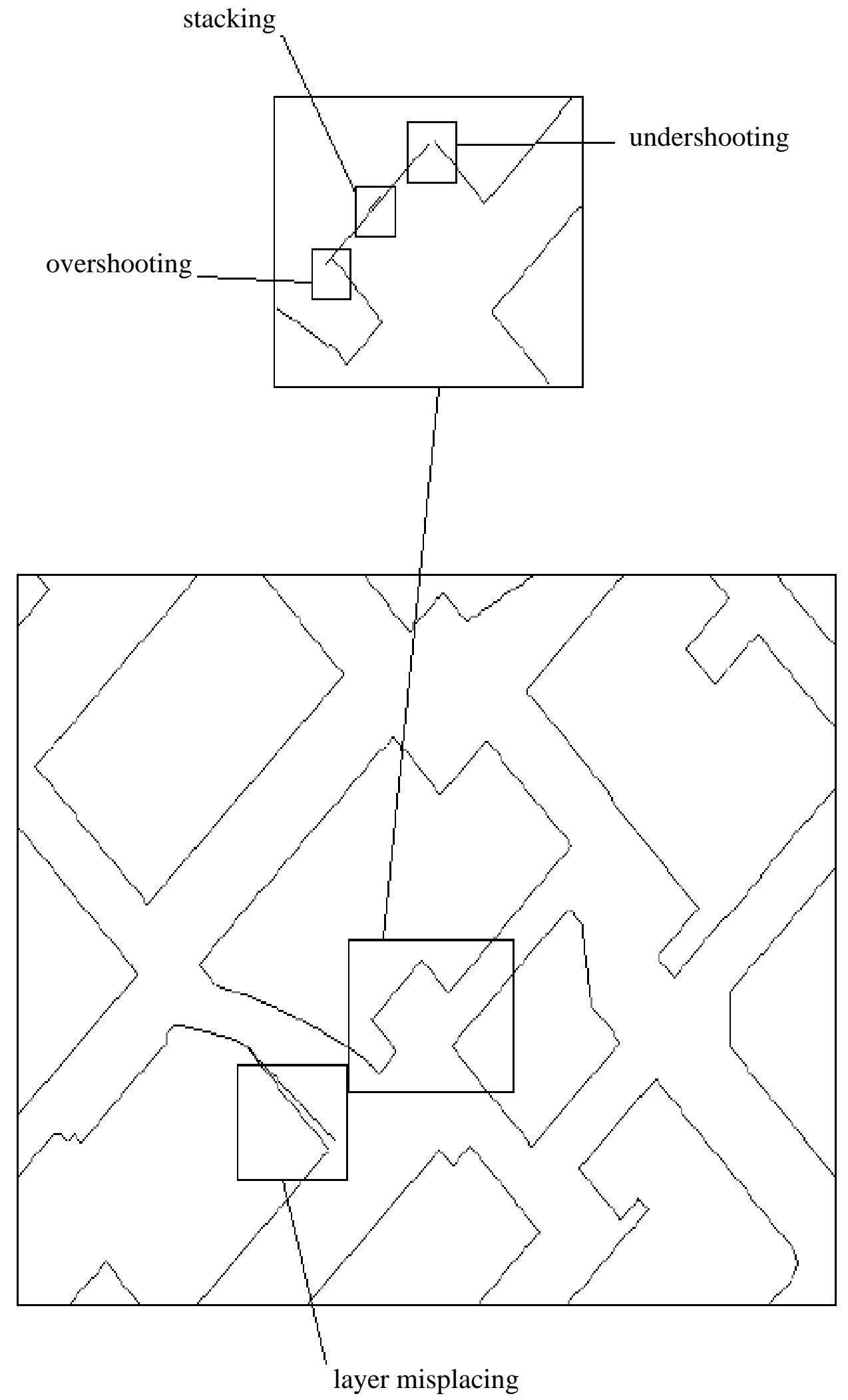

Fig. 2 




Fig. 3 


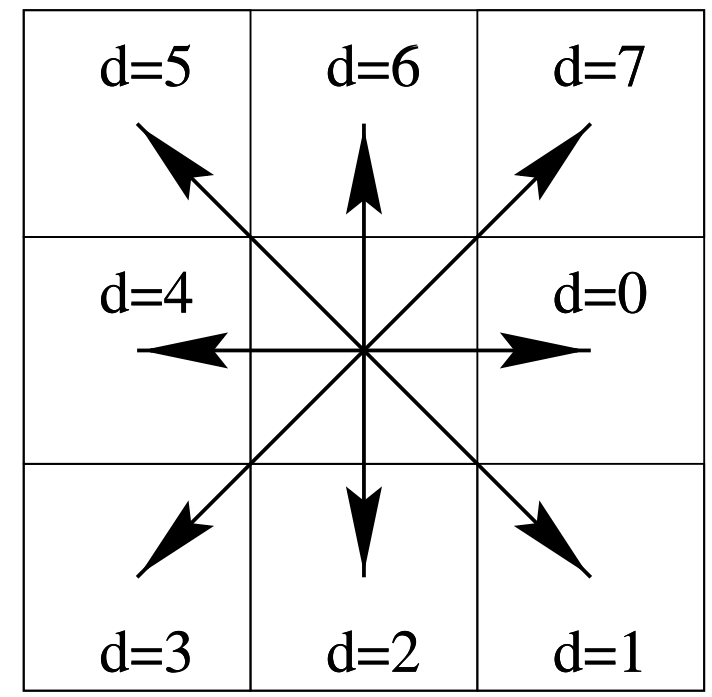

Fig. 4 

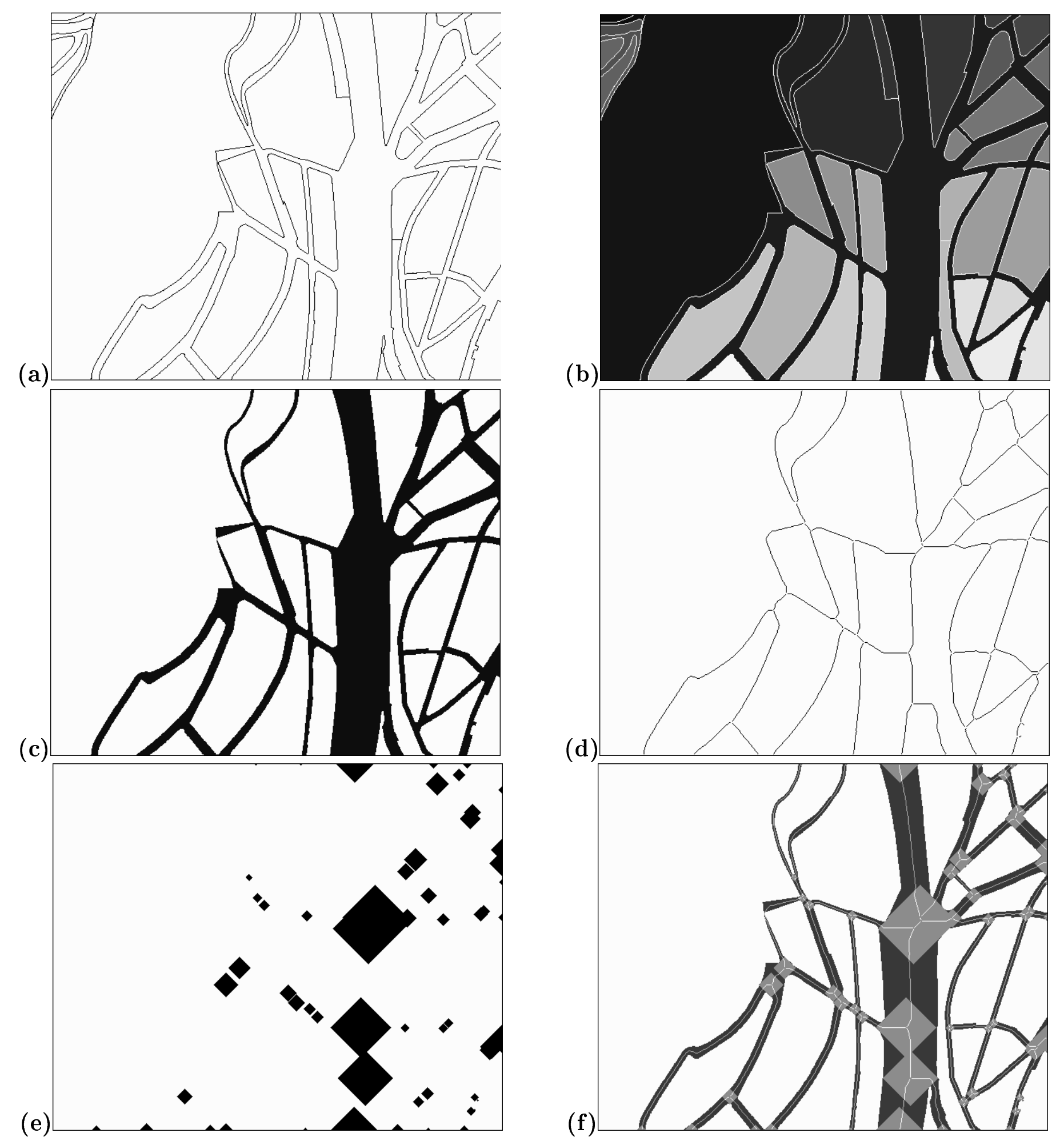

(d)

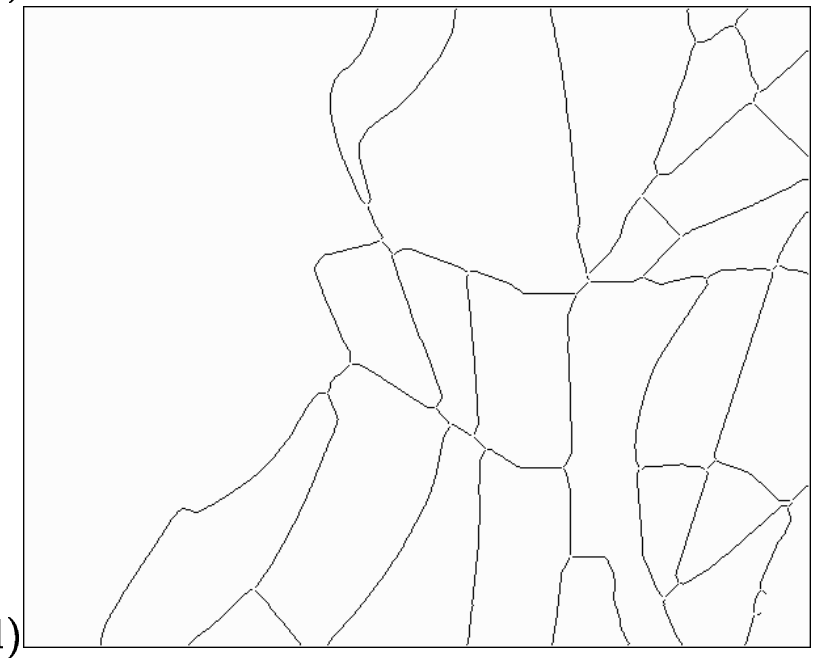

(f)

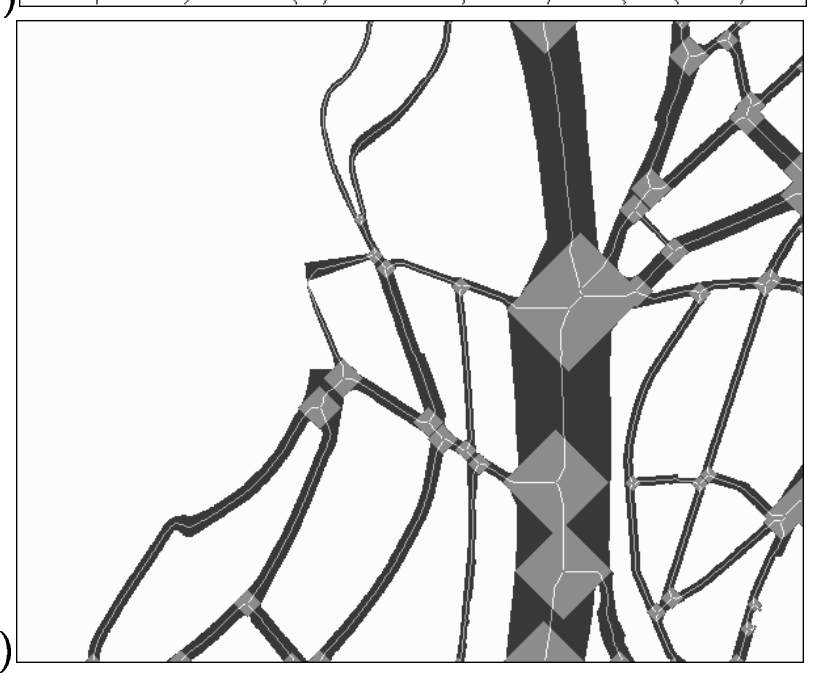

Fig. 5 

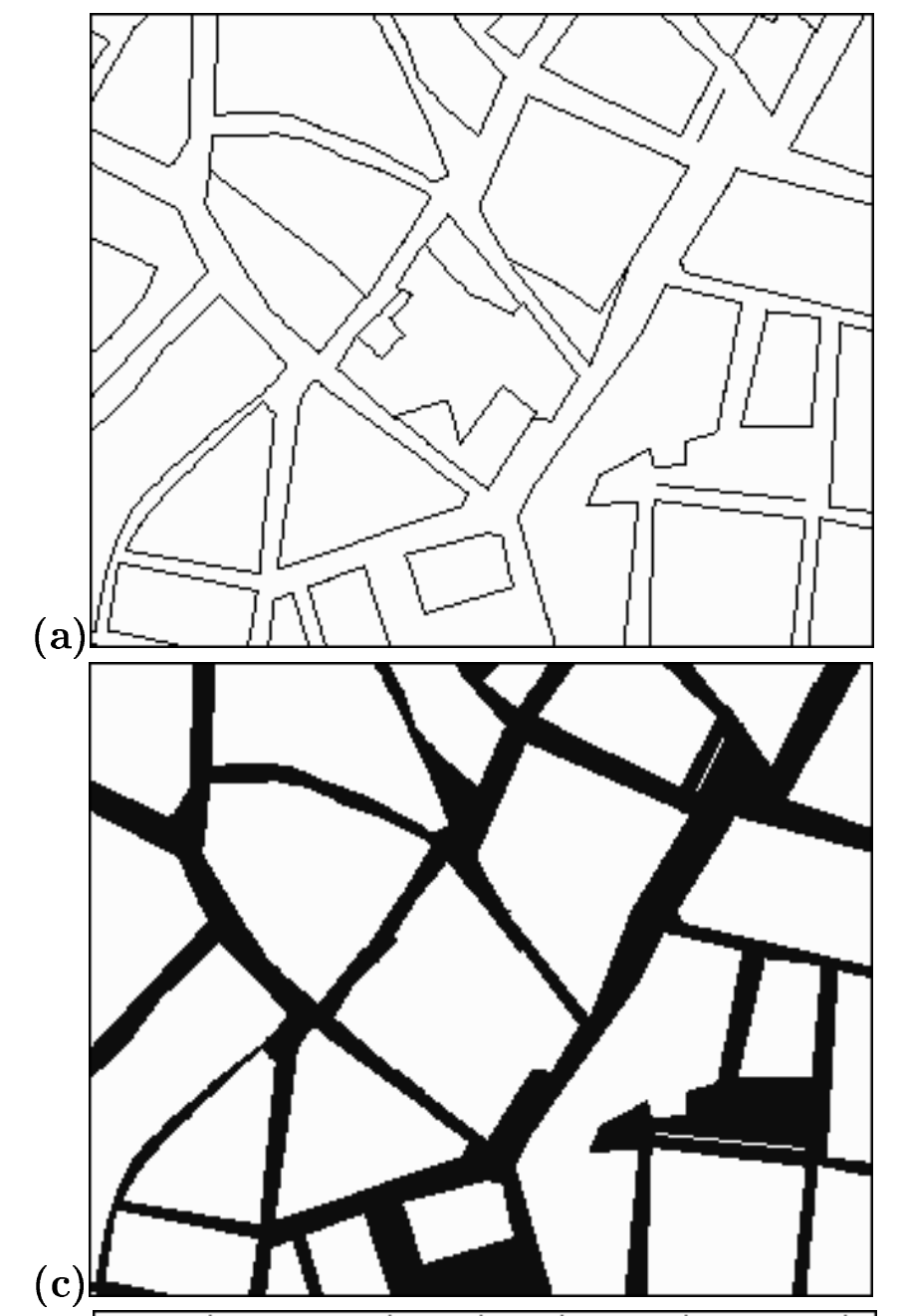

(b)
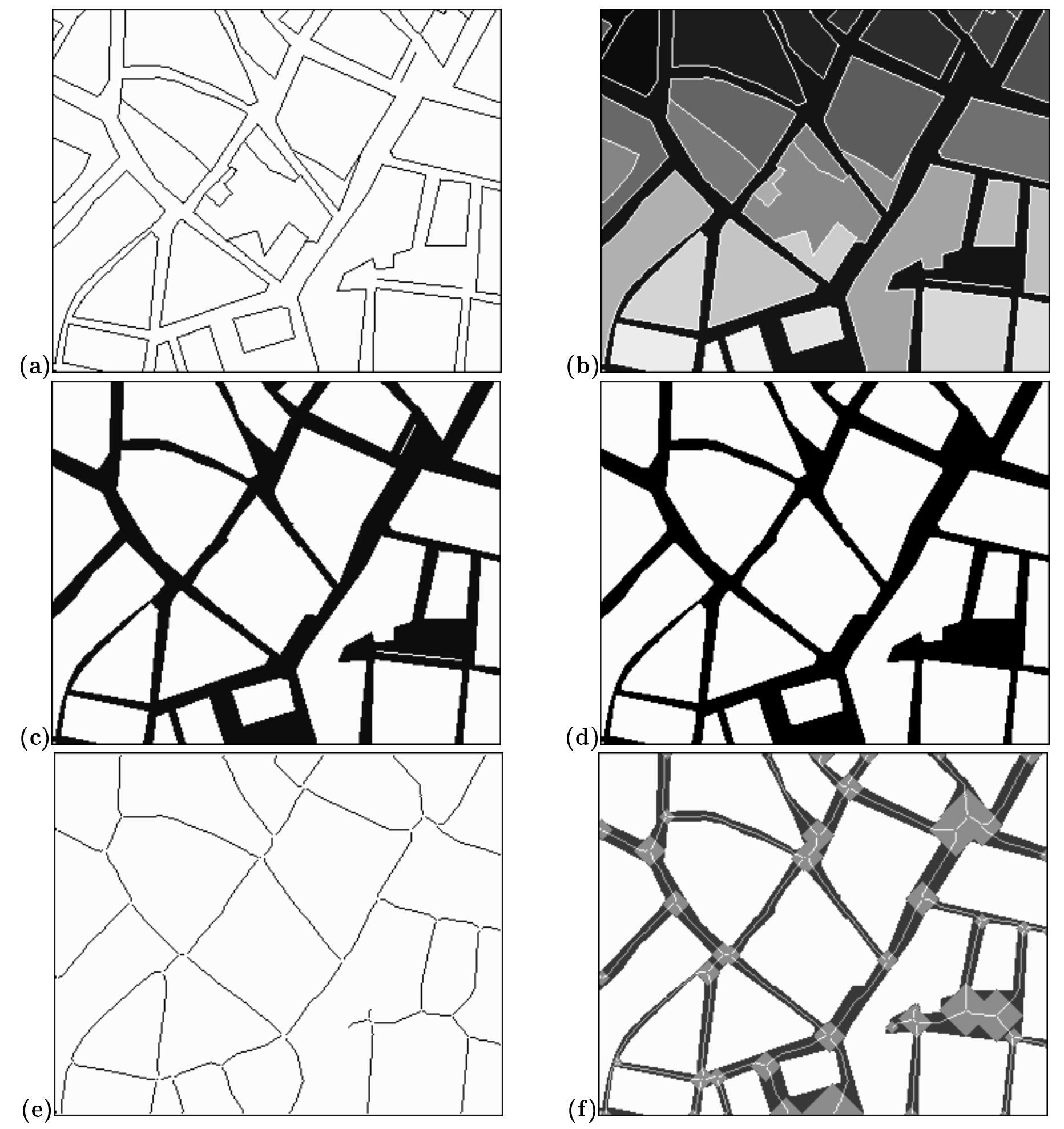

(d)

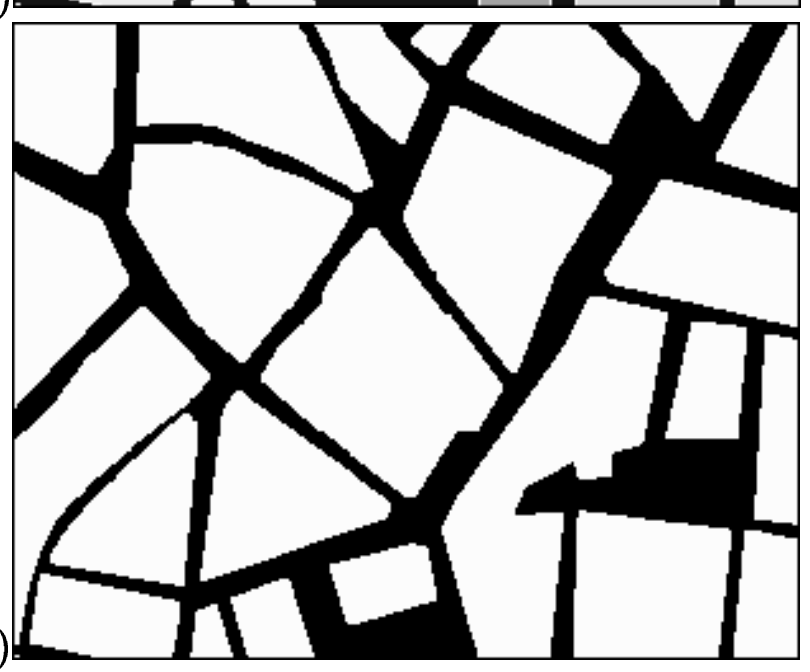

(f)

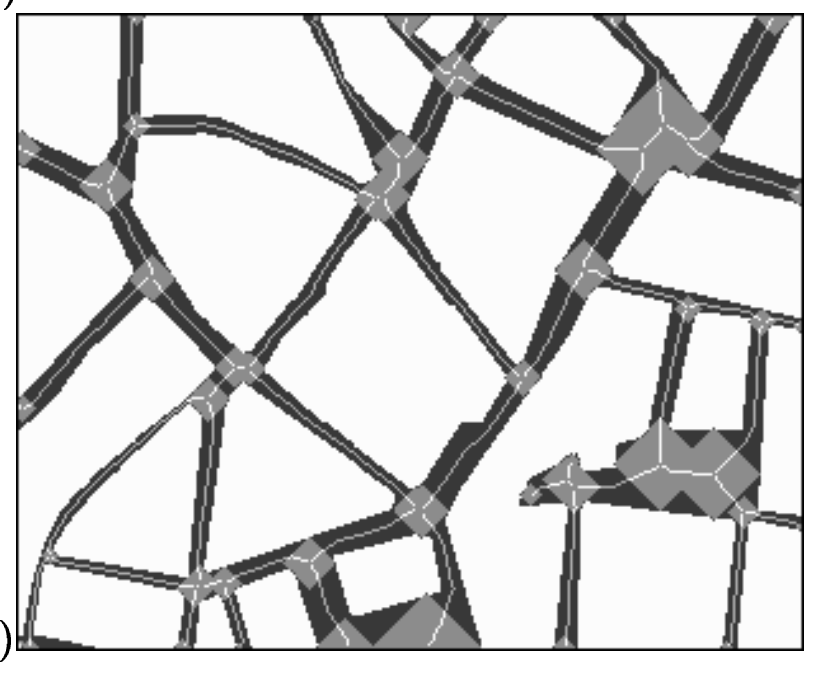

Fig. 6 

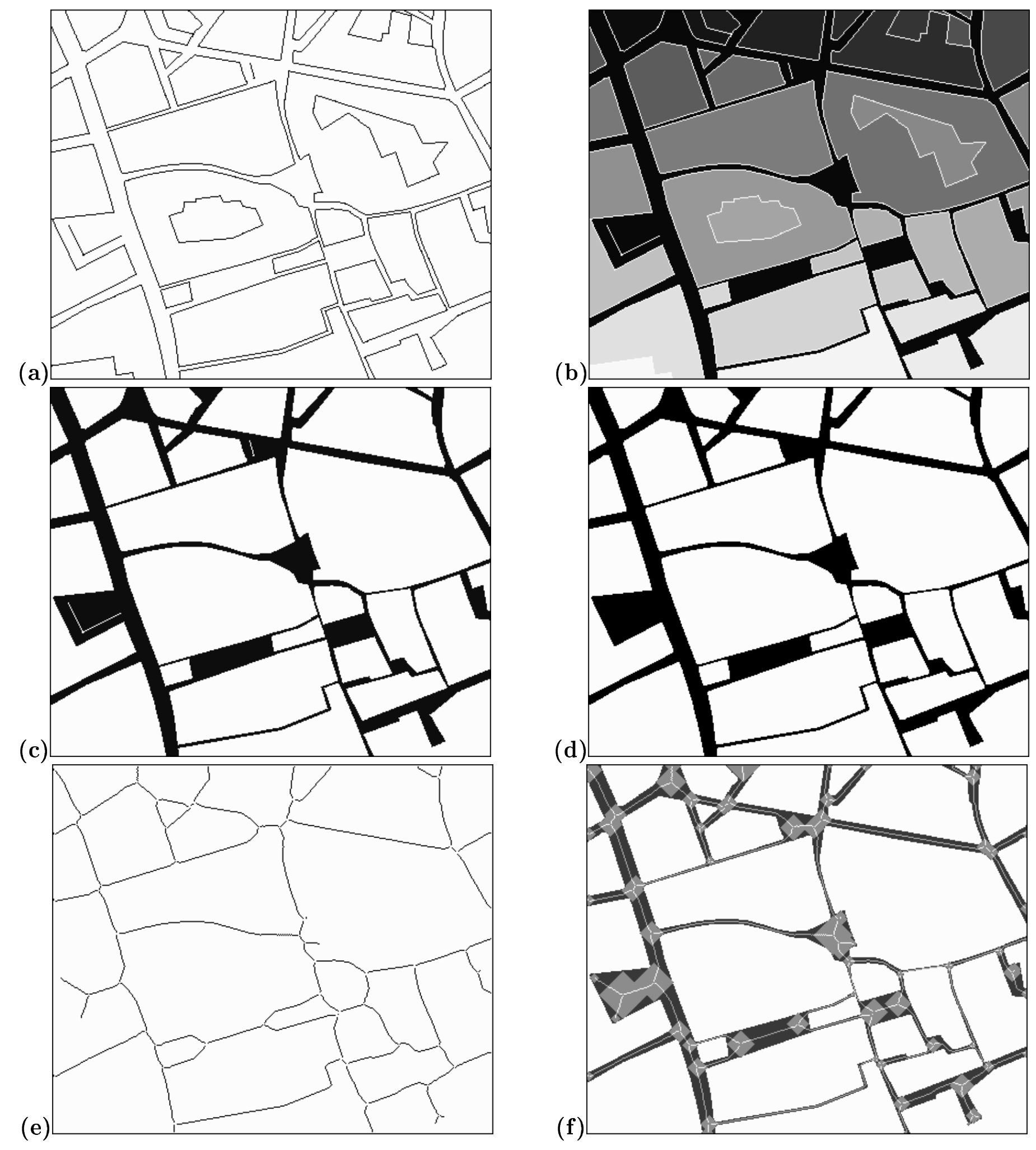

Fig. 7 